\title{
Wrapped Generalized Gompertz distribution: an Application to Ornithology
}

\author{
Shongkour Roy ${ }^{1 *}$ and Mian Arif Shams Adnan²
}

${ }^{1}$ Center for Nutrition and Food Security (CNFS), International Center for Diahorreal Diseases Research (ICDDRB), Mohakhali, Dhaka, Bangladesh ${ }^{2}$ Department of Statistics, Jahangirnagar University, Dhaka, Bangladesh

\begin{abstract}
In many scientific fields such as biology (orientation of birds), geology (orientations of feldspar laths) and meteorology (wind direction and ozone concentration), data occur as angular forms. In this paper, a class of wrapped distribution called wrapped generalized Gompertz distributions are introduced (WGG). Characteristic function and fundamental properties of this distribution are described. Some theorems that relate the distribution to some other circular distributions are established. Applications to density estimation and goodness-of-fit tests are used to analyze data on the heading of orientation of the nest of noisy scrub birds, and it is shown that the model fits much better than some other existing circular symmetric and non-symmetric models.
\end{abstract}

Keywords: Directional data; Generalized Gompertz distribution; Trigonometric moments; Wrapped distribution; Watson statistics

\section{Introduction}

Levy [1] proposed a novel method for introducing a wrapped variable to the symmetric and non-symmetric distribution, which is known in the literature as a wrapped distribution. Several authors have done the extensive work on introducing wrapped distribution such as wrapped exponential, wrapped Laplace, wrapped Cauchy, wrapped Chi-square and wrapped weighted exponential distribution. Moreover, their statistical properties and inference procedures have been investigated thoroughly in the literature; for example Jammalamadaka and Kozubowski [2,3], Levy [1], Roy and Adnan [4-6]. However, it seems that no attempts were made to implement Levy [1] idea for non-symmetric distribution, in case of wrapped generalized Gompertz distributions, which produce equally interesting circular models.

For the real line, Willemse and Kaas [7] first introduced the generalized Gompertz distribution and its different properties. Furthermore, they showed that generalized Gompertz distribution posses some good properties and can be used as a good fit to survival model in biology, compared to the other popular distributions such as exponential, Gamma or logistic distributions.

In this paper, a class of wrapped generalized Gompertz distribution is introduced. This class of distribution is found with wrapping a generalized Gompertz distribution, introduced by Willemse and Kaas [7] and Coelho [8] around the circumference of a unit circle, and GG distribution defined as follows: a random variable $\mathrm{X}$ is said to have a generalized Gompertz distribution with a shape parameter $b>0$, scale parameter $c>0$, and location parameter $-\infty<a<\infty$, denoted by GG (a, b, c), if it probability density function (PDF) is given as follows:

$$
f_{X}(x)=\frac{1}{b \Gamma(c)} \exp \left(c \frac{x-a}{b}-e^{\frac{x-a}{b}}\right),-\infty<x<\infty
$$

And cumulative density function (CDF)

$$
F_{X}(x)=1-\frac{\Gamma\left(c, \exp \left(\frac{x-a}{b}\right)\right)}{\Gamma(c)},-\infty<x<\infty
$$

Where, $\Gamma\left(c, \exp \left(\frac{x-a}{b}\right)\right)$ denotes the upper gamma function

$$
\Gamma\left(c, \exp \left(\frac{x-a}{b}\right)\right)=\int_{\exp \left(\frac{x-a}{b}\right)}^{\infty} v^{c-1} e^{-v} d v \text {, while } \Gamma(c)=\Gamma(c, 0)
$$

The main objective of this article is to introduce a class of distribution that wrapped generalized Gompertz distribution, and provide an application in ornithology.

The rest of the paper is organized as follows: Sect. 3.1 provides the probability and cumulative density function of the wrapped generalized Gompertz distribution. Sect. 3.2 makes explicit the characteristic function and the fundamental properties with related parameters Maximum likelihood estimation is given in Sect. 3.5. In Sect. 3.6, an application in ornithology has been discussed briefly, illustrating the modeling potential of wrapped generalized Gompertz distribution. Finally, the conclusion of the paper appears in Sect. 4.

Figure 1 depicts some wrapped generalized Gompertz densities.

\section{Derivation of the density}

In this section, the definition of the wrapped WGG distribution was introduced, denoted by WGG (a, b, c), and cumulative density function of the same distribution.

$$
\text { Let us consider } \theta=\theta(x)=X(\bmod 2 \pi)
$$

Then, $\theta$ is wrapped (around the circle) or a generalized Gompertz circular random variable, that for $\theta \in(0,2 \pi)$ has the probability density function

$$
\begin{aligned}
& g(\theta)=\sum_{k=-\infty}^{\infty} f_{x}(\theta+2 k \pi) \\
& g(\theta)=\frac{1}{b \Gamma(c)} \sum_{k=-\infty}^{\infty} \exp \left(c \frac{\theta+2 \pi k-a}{b}-e^{\frac{\theta+2 \pi k-a}{b}}\right) ; \\
& \theta \in[0,2 \pi), b>0, c>0, \theta+2 \pi k>a, a \in[0,2 \pi)
\end{aligned}
$$

*Corresponding author: Shongkour Roy, Center for Nutrition and Food Security (CNFS), International Center for Diahorreal Diseases Research (ICDDRB), Mohakhali, Dhaka-1212, Bangladesh, E-mail: sankar1604@gmail.com

Received August 30, 2012; Accepted October 29, 2012; Published November 03, 2012

Citation: Roy S, Shams Adnan MA (2012) Wrapped Generalized Gompertz distribution: an Application to Ornithology. J Biom Biostat 3:153. doi:10.4172/21556180.1000153

Copyright: (c) 2012 Roy S, et al. This is an open-access article distributed under the terms of the Creative Commons Attribution License, which permits unrestricted use, distribution, and reproduction in any medium, provided the original author and source are credited. 
Citation: Roy S, Shams Adnan MA (2012) Wrapped Generalized Gompertz distribution: an Application to Ornithology. J Biom Biostat 3:153. doi:10.4172/2155-6180.1000153

which is the probability density function of wrapped generalized Gompertz distribution. The random variable $\theta$ has a wrapped generalized Gompertz distribution, with a parameter and the above PDF as follows: $\theta \sim W G G(a, b, c)$.

Figure 1 depicts the probability density function of the wrapped generalized Gompertz distribution.

Now, according to Mardia and Jupp [9], Jammalamadaka and SenGupta [10] cumulative density function of wrapped generalized Gompertz distribution can be obtained as

$$
G(\theta)=\sum_{m=0}^{\infty} F(\theta+2 \pi k)-F(2 \pi k) ; 0 \leq \theta<2 \pi .
$$

Using the immediate equation, the cumulative density function (CDF) of wrapped generalized Gompertz distribution could be got.

$$
\begin{aligned}
& G(\theta)=\sum_{k=-\infty}^{\infty}\left(\left[1-\frac{\Gamma\left(c, \exp \left(\frac{\theta+2 \pi k-a}{b}\right)\right)}{\Gamma(c)}\right]-\left[1-\frac{\Gamma\left(c, \exp \left(\frac{2 \pi k-a}{b}\right)\right)}{\Gamma(c)}\right]\right) \\
& =\sum_{k=-\infty}^{\infty} \frac{1}{\Gamma(c)}\left[\Gamma\left(c, \exp \left(\frac{2 \pi k-a}{b}\right)\right)-\Gamma\left(c, \exp \left(\frac{\theta+2 \pi k-a}{b}\right)\right)\right] .
\end{aligned}
$$

\section{Fundamental properties of the density}

The trigonometric moment of order, p, for a wrapped circular distribution corresponds to the value of the characteristic function of the unwrapped r.v. X say $\phi_{x}(t)$ at the integer value $\mathrm{p}[10]$.

$$
\phi(p)=\phi_{x}(t) \text {. }
$$

On the real line, the characteristic function of the generalized Gompertz distribution is

Use the fact that $X \sim b \ln Y+a$ for a Gamma(c, 1) random variable Y $\phi_{x}(t)=E\left(e^{i t x}\right)=E\left[e^{i t(b \ln Y+a)}\right]$

Wrapped generalized Gompertz distribution

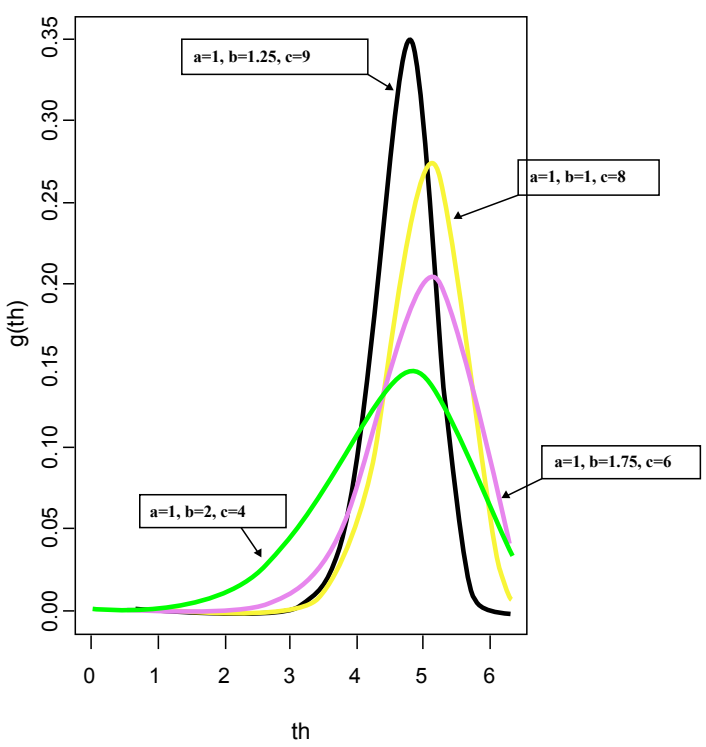

Figure 1: Some wrapped generalized Gompertz densities

$$
=e^{i t a} E\left(y^{i t b}\right)=e^{i t a} \int_{0}^{\infty} y^{i t b+c-1} e^{-y} d y=e^{i t a} \frac{\Gamma(i t b+c)}{\Gamma(c)} .
$$

Hence, using the Eq. (5), the characteristic function of the wrapped generalized Gompertz distribution is obtained as

$$
\begin{aligned}
& \phi(p)=e^{i p a} \frac{\Gamma(i p b+c)}{\Gamma(c)} ; p= \pm 1, \pm 2, \pm 3, \ldots, \text { where } i=(-1)^{\frac{1}{2}} \\
& \phi(p)=\rho_{p} e^{i \mu_{p}} \\
& \text { Where } \rho_{p}=\frac{\Gamma(i p b+c)}{\Gamma(c)}, \mu_{p}=p a .
\end{aligned}
$$

\section{Trigonometric moments and related parameters}

Using the definition of the trigonometric moment,

$$
\phi_{p}=\alpha_{p}+i \beta_{p} ; p= \pm 1, \pm 2, \ldots
$$

And therefore, the non-central trigonometric moments of the respective distribution are:

$\alpha_{p}=\rho_{p} \cos \left(\mu_{p}\right)$ And $\beta_{p}=\rho_{p} \sin \left(\mu_{p}\right)$.

So, the equation comes to be as $\alpha_{p}=\frac{\Gamma(i p b+c)}{\Gamma(c)} \cos (p a)$ and $\beta_{p}=\frac{\Gamma(i p b+c)}{\Gamma(c)} \sin (p a)$.

Now, according to Jammalamadaka and SenGupta [10], an alternative expression for the PDF of the wrapped generalized Gompertz distribution can be obtained, using the trigonometric moments as

$$
g(\theta)=\frac{1}{2 \pi}\left[1+2 \sum_{p=1}^{\infty}\left(\alpha_{p} \cos p \theta+\beta_{p} \sin p \theta\right)\right] ; \theta \in[0,2 \pi) .
$$

As such substituting the value of $\alpha_{p}$ and $\beta_{p}$ in the immediate above equation, the equation got is:

$$
\begin{aligned}
& g(\theta)=\frac{1}{2 \pi}\left[1+2 \sum_{p=1}^{\infty}\left(\frac{\Gamma(i p b+c)}{\Gamma(c)} \cos (p a) \cos p \theta+\frac{\Gamma(i p b+c)}{\Gamma(c)} \sin (p a) \sin p \theta\right)\right] \\
& \quad=\frac{1}{2 \pi}\left[1+2 \sum_{p=1}^{\infty} \frac{\Gamma(i p b+c)}{\Gamma(c)}(\cos (p a) \cos p \theta+\sin (p a) \sin p \theta)\right] \\
& \therefore g(\theta)=\frac{1}{2 \pi}\left[1+2 \sum_{p=1}^{\infty} \frac{\Gamma(i p b+c)}{\Gamma(c)} \cos p(\theta-a)\right] ; \quad \theta \in[0,2 \pi], b>0, \theta+2 \pi k>a,
\end{aligned}
$$
$a \in[0,2 \pi]$

This is the alternative PDF of wrapped generalized Gompertz distribution.

Now, the central trigonometric moment is defined as

$\bar{\alpha}_{p}=\rho_{p} \cos \left(\mu_{p}-p \mu_{1}\right)$ And $\bar{\beta}_{p}=\rho_{p} \sin \left(\mu_{p}-p \mu_{1}\right)$. will be

Thus, the central trigonometric moments of the same distribution

Therefore, $\bar{\alpha}_{p}=\frac{\Gamma(i p b+c)}{\Gamma(c)} \cos (a p-p a)$ and $\overline{\beta_{p}}=\frac{\Gamma(i p b+c)}{\Gamma(c)} \sin (a p-p a)$

$\therefore \bar{\alpha}_{p}=\frac{\Gamma(i p b+c)}{\Gamma(c)}$ And $\overline{\beta_{p}}=0$.

The resultant length, $\rho=\rho_{1}$ thus $\rho=\frac{\Gamma(i b+c)}{\Gamma(c)}$,

The Mean direction, $\mu=\mu_{1}$ so that $\mu_{1}=a$

The circular variance is

$V_{0}=1-\rho, V_{0}=1-\frac{\Gamma(i b+c)}{\Gamma(c)}$,

And the circular standard deviation is

$\sigma_{0}=\sqrt{-2 \log \left(1-V_{0}\right)}$ 


$$
\begin{aligned}
& =\sqrt{-2 \log (1-1+\rho)} \\
& =\sqrt{-2 \log \frac{\Gamma(i b+c)}{\Gamma(c)}}
\end{aligned}
$$

The kurtosis is $\varsigma_{2}^{0}=\frac{\bar{\alpha}_{2}-\left(1-V_{0}\right)^{4}}{V_{0}^{2}}$ given by

$$
\varsigma_{2}^{0}=\frac{\frac{\Gamma(2 i b+c)}{\Gamma(c)}-\left(\frac{\Gamma(i b+c)}{\Gamma(c)}\right)^{4}}{\left(1-\frac{\Gamma(i b+c)}{\Gamma(c)}\right)^{2}}
$$

Since from (7) for $\mathrm{p}=2, \bar{\alpha}_{2}=\frac{\Gamma(2 i b+c)}{\Gamma(c)}$.

Theorem: The difference between two wrapped generalized Gompertz distributed angular variables are also a wrapped generalized Logistic distribution.

Proof: Let us consider two independent angular variables with generalized Gompertz distributions are $\theta_{1}$ and $\theta_{2}$ its difference $\theta_{1}-\theta_{2}$.

i.e. $\theta_{1} \sim W G G\left(a_{1}, b_{1}, c_{1}\right)$ and $\theta_{2} \sim W G G\left(a_{2}, b_{2}, c_{2}\right)$, providing in fact a generalization of the wrapped logistic distribution, assuming $a_{1}=a, a_{2}=0$, equal parameters $c 1$ and $c 2$, the characteristic function of $\theta_{1}-\theta_{2}$, with

$$
\begin{aligned}
& \theta_{1} \sim W G G\left(a, b, c_{1}\right) \text { and } \theta_{2} \sim W G G\left(0, b, c_{2}\right) \text { is } \\
& \phi_{\theta_{1}-\theta_{2}}(p)=\phi_{\theta_{1}}(p) \phi_{\theta_{2}}(-p)=e^{i a p} \frac{\Gamma\left(c_{1}+i b p\right) \Gamma\left(c_{2}-i b p\right)}{\Gamma\left(c_{1}\right) \Gamma\left(c_{2}\right)}
\end{aligned}
$$

Since, we know that $c h$. $f$. satisfies uniquely any distribution function, thus $\theta_{1}-\theta_{2}$ is also wrapped generalized Logistic distribution [11].

\section{Wrapped logistic distribution}

In special case, wherein (8) $a_{1}=a_{2}=0, b_{1}=b_{2}=1$ and $c_{1}=c_{2}=1$, the difference between two standard wrapped Gompertz angular variables is got, such an angular variable has a wrapped logistic distribution, i.e.

$$
\begin{aligned}
\phi_{\theta_{1}-\theta_{2}}(p) & =e^{i a p} \frac{\Gamma(1+i p) \Gamma(1-i p)}{\Gamma(1) \Gamma(1)} \\
& =e^{i a p} B(1+i p, 1-i p) \text { For }|i p|<1
\end{aligned}
$$

which is the ch.f. of wrapped logistic distribution, and its density function is given below

$$
g(\theta)=\frac{1}{2 \pi}\left[1+2\left(\sum_{p=1}^{\infty} \beta(1-i p, i+i p) \cos (\theta-a)\right)\right] ; \theta \in[0,2 \pi), a \in[0,2 \pi) .
$$

\section{Maximum Likelihood Estimation}

In this section, the maximum likelihood estimators of the unknown parameters $(\mathrm{a}, \mathrm{b}, \mathrm{c})$ of the $W G G(\mathrm{a}, \mathrm{b}, \mathrm{c})$ are derived. Assume $\theta_{1}, \theta_{2}, \ldots, \theta_{n}$ be a random sample of size $\mathrm{n}$ from $W G G(\mathrm{a}, \mathrm{b}, \mathrm{c})$, then the likelihood functions $l$ is [12]

$$
l=\prod_{i=1}^{n} g\left(\theta_{i} ; a, b, c\right) .
$$

Substituting from (9) into (4) to be get

$$
\begin{aligned}
& l=\prod_{i=1}^{n} \frac{1}{b \Gamma(c)} \sum_{k=-\infty}^{\infty} \exp \left(c \frac{\theta+2 \pi k-a}{b}-e^{\frac{\theta+2 \pi k-a}{b}}\right), \\
& l=\left[\frac{1}{b \Gamma(c)}\right]^{n}\left(\sum_{k=-\infty}^{\infty} e^{\sum_{i=1}^{n}\left(\theta_{i}+2 \pi k-a\right)} b-\sum_{k=-\infty}^{\infty} e^{e_{i=1}^{n} b\left(\theta_{i}+2 \pi k-a\right)}\right),
\end{aligned}
$$

$$
l=\left[\frac{1}{b \Gamma(c)}\right]^{n}\left[\left(e^{-\frac{n a c}{b}} e^{\frac{c \sum_{i=1}^{n} \theta_{i}}{b}} \sum_{k=-\infty}^{\infty} e^{\frac{2 \pi n c k}{b}}\right)-\left(e^{-\frac{n a}{b}} e^{e^{\sum_{i=1}^{n} \theta_{i}}} \sum_{k=-\infty}^{\infty} e^{e^{\frac{2 \pi n k}{b}}}\right)\right],
$$

The $\log$ likelihood function $L=\ln (l)$ is given by

$$
L=-n \log (b)-n \log (\Gamma(c))-\frac{n a c}{b}+\frac{c \sum_{i=1}^{n} \theta_{i}}{b}+\sum_{k=-\infty}^{\infty} \frac{2 \pi n c k}{b}-e^{\frac{\sum_{i=1}^{n} \theta_{i}}{b}}-e^{-\frac{n a}{b}}-\sum_{k=-\infty}^{\infty} e^{\frac{2 \pi n k}{b}} .
$$

The first partial derivatives of the $\log$ likelihood $L$ with respect to $a, b$ and $c$ are obtained as follows

$$
\begin{aligned}
& \frac{\partial L}{\partial a}=-\frac{n c}{b}+\frac{n}{b} e^{-\frac{n a}{b}}, \\
& \frac{\partial L}{\partial b}=-\frac{n}{b}+\frac{n a c}{b^{2}}-\frac{c \sum_{i=1}^{n} \theta_{i}}{b^{2}}-\sum_{k=-\infty}^{\infty} \frac{2 \pi n c k}{b^{2}}+\frac{\sum_{i=1}^{n} \theta_{i}}{b^{2}} e^{\frac{\sum_{i=1}^{n} \theta_{i}}{b}}-\frac{n a}{b^{2}} e^{-\frac{n a}{b}}+\frac{2 \pi n}{b^{2}} \sum_{k=-\infty}^{\infty} K e^{\frac{2 \pi n k}{b}}, \\
& \frac{\partial L}{\partial c}=-\frac{n}{\Gamma(c)} \Psi(\Gamma(c))-\frac{n a}{b}+\frac{\sum_{i=1}^{n} \theta_{i}}{b}+\sum_{k=-\infty}^{\infty} \frac{2 \pi n k}{b} .
\end{aligned}
$$

The likelihood equations can be obtained by setting the first partial derivatives of $L$ to zeros. That is, the likelihood equations take the following form:

$$
\begin{aligned}
& -\frac{n c}{b}+\frac{n}{b} e^{-\frac{n a}{b}}=0, \\
& -\frac{n}{b}+\frac{n a c}{b^{2}}-\frac{c \sum_{i=1}^{n} \theta_{i}}{b^{2}}-\sum_{k=-\infty}^{\infty} \frac{2 \pi n c k}{b^{2}}+\frac{\sum_{i=1}^{n} \theta_{i}}{b^{2}} e^{\frac{\sum_{i=1}^{n} \theta_{i}}{b}}-\frac{n a}{b^{2}} e^{-\frac{n a}{b}}+\frac{2 \pi n}{b^{2}} \sum_{k=-\infty}^{\infty} K e^{\frac{2 \pi n k}{b}}=0, \\
& -\frac{n}{\Gamma(c)} \Psi(\Gamma(c))-\frac{n a}{b}+\frac{\sum_{i=1}^{n} \theta_{i}}{b}+\sum_{k=-\infty}^{\infty} \frac{2 \pi n k}{b}=0 .
\end{aligned}
$$

To derive the MLEs of the unknown parameters $a, b$, and $c$, the above non-linear system of equations in $a, b$, and $c$ has to be solved.

From (13), the MLE of as a function of estimators of the rest of the parameters $(\hat{b}, \hat{c})$ as $\hat{a}$ can be got [13].

\section{An application in ornithology}

The following application shows the effectiveness of the WGG model when applied to some data from a real problem. Such data frequently form orientation in biology [14-18]. The data set was taken from Fisher [19]. The main goal is to provide for monitoring the orientations of the nest of noisy scrub birds, along the bank of a creek bed. The species of birds in this region are more important for the biodiversity. This study is important due to the growing evidence of the vulnerability of the noisy scrub birds to chance in biodiversity. To demonstrate the modeling behavior of the wrapped generalized Gompertz distribution, we consider nest orientations of noisy of scrub birds, discussed by Fisher [19]. For the orientation of the nests of 50 noisy scrub birds along the bank of a creek bed, the data (degree) are as given below:

$160^{\circ}, 145^{\circ}, 225^{\circ}, 230^{\circ}, 295^{\circ}, 295^{\circ}, 140^{\circ}, 140^{\circ}, 140^{\circ}, 205^{\circ}, 215^{\circ}, 135^{\circ}$, $110^{\circ}, 240^{\circ}, 230^{\circ}, 250^{\circ}, 30^{\circ}, 215^{\circ}, 215^{\circ}, 135^{\circ}, 110^{\circ}, 240^{\circ}, 105^{\circ}, 125^{\circ}, 125^{\circ}$, $130^{\circ}, 160^{\circ}, 105^{\circ}, 90^{\circ}, 130^{\circ}, 200^{\circ}, 240^{\circ}, 105^{\circ}, 125^{\circ}, 125^{\circ}, 125^{\circ}, 130^{\circ}, 160^{\circ}$, $160^{\circ}, 250^{\circ}, 200^{\circ}, 200^{\circ}, 240^{\circ}, 240^{\circ}, 240^{\circ}, 250^{\circ}, 250^{\circ}, 250^{\circ}, 140^{\circ}, 140^{\circ}$

Assuming a symmetric wrapped generalized Gompertz model (with $b=1$ ), the first step is to find the maximum likelihood estimates of the parameters a and $c$ for a WGG distribution. This was carried out with maple in the following results of radian measure over $(0,2 \pi)$. Again 
Citation: Roy S, Shams Adnan MA (2012) Wrapped Generalized Gompertz distribution: an Application to Ornithology. J Biom Biostat 3:153. doi:10.4172/2155-6180.1000153

\begin{tabular}{|c|c|c|c|c|}
\hline Distributions & MLEs & Log-likelihood & AIC & BIC \\
\hline$v M(\mu, k)$ & $(3.0640,1.3090)$ & -22.8794 & 57.7589 & 69.2310 \\
\hline$W N(\mu, \rho)$ & $(3.0900,0.5773)$ & -22.9907 & 57.9814 & 69.4535 \\
\hline$W C(\mu, \rho)$ & $(2.7420,0.4080)$ & -23.1427 & 58.3054 & 69.7776 \\
\hline$W G G(a, c)$ & $(4.4600,0.0570)$ & -22.6904 & 57.2189 & 68.6910 \\
\hline
\end{tabular}

Table 1: Summary of fits for the noisy scrub bird's data.

using $\mathrm{R}$ with circstat package, the MLE of the other circular models is fit therein, i.e. vonMises (vM), wrapped Cauchy distribution (WC) and wrapped normal $(\mathrm{WN})$. Table 1 provides the relevant numerical summaries for the four fits.

To check the goodness of fit of the distributions, WGG, vM, WC and $\mathrm{WN}$, both the kuiper and Watson statistics for each model are computed. These tests may be found in reference Jammalamadaka and SenGupta [10]. Using the values of $\hat{a}$ and $\hat{c}$ in table 1, the kuiper statistic and the Watson statistic for a WGG distribution has the values, $V_{50}=6.5548$ and $W_{50}^{2}=3.7983$, respectively [20]. Again using the values of $\hat{\mu}$ and $\hat{k}$ in table 1 , the kuiper statistic and the Watson statistic for a vM distribution has the values, $V_{50}=6.9323$ and $W_{50}^{2}=3.9240$ respectively. For WC and WN distribution, the kuiper and Watson statistic values, $V_{50}=6.9096, W_{50}^{2}=3.7995$ and $V_{50}=6.8604, W_{50}^{2}=3.8805$, respectively. The smaller value of the both statistic, AIC and BIC for a WGG distribution indicate a better fit. Thus, it is confirmed that the wrapped generalized Gompertz distribution provides a superior model for these data than the vonMises, wrapped Cauchy and wrapped normal distributions.

\section{Conclusions}

In this paper, an expression for the probability density function of wrapped generalized Gompertz distribution is suggested. Some of the other wrapped distributions have also been unfolded through proper specification of the parameters. The alternative form of the PDF of the same distribution is also obtained using trigonometric moments. A class of basic properties is also found here. Modules programmed in or with circstat package, of maple- 12 for the concern computations and graphics are available. Finally, effectiveness of this model with real data from ornithology was depicted.

\section{Acknowledgments}

The author thanks the editor and two anonymous referees for thoughtful comments and corrections. The paper have been substantially improved due to their constructive feedbacks.

\section{References}

1. Levy PL (1939) Addition des variables al'eatoires d'efinies sur une circonf'erence. Bull Soc Math 67: 1-41.

2. Jammalamadaka SR, Kozubowski TJ (2004) New families of wrapped distributions for modelling skew circular data. Commun Stat Theory Methods 33: $2059-2074$

3. Jammalamadaka SR, Kozubowski TJ (2003) A new family of circular models: the wrapped Laplace distributions. Adv Appl Stat 3: 77-103.

4. Adnan MAS, Roy S (2011) Wrapped Chi-square distribution. J of Appl Stat Sci 18: $307-317$

5. Roy S, Adnan MAS (2012) Wrapped weighted exponential distributions. Stat Prob Lett 82: 77-83.

6. Roy S, Adnan MAS (2010) Wrapped Three parameter Gamma distribution JSM Vancouver, British Columbia, Canada.

7. Willemse WJ, Kaas R (2006) Rational reconstruction of frailty-based mortality models by a generalisation of Gompertz' law of mortality. Insu: Math Econ.

8. Coelho CA (2007) The wrapped Gamma distribution and wrapped sums and linear combinations of independent Gamma and Laplace distributions. Stat Theory and Prac 1: 1-29.
9. Mardia KV, Jupp PE (1972) Directional Statistics. John Wiley \& Sons, Academic press, New York.

10. Jammalamadaka SR, SenGupta A (2001) Topics in circular Statistics. World scientific, Singapore.

11. Dattatreya RAV, Ramabhadra SI, Girija SVS (2007) On wrapped version of some life testing models. Commun Stat Theory Methods 36: 2027-2035.

12. Gatto R, Jammalamadaka SR (2002) Inference for wrapped symmetric -stable circular models. Sankhya (In press).

13. Self SG, Liang KY (1987) Asymptotic properties of maximum likelihood estimators and likelihood ratio tests under nonstandard conditions. J Am Stat Assoc 82: 605-610.

14. Jander $R$ (1957) The optical directional orientation of the red wood ant (Formica Rufa L.). Cf Z Physiol 40: 162-238.

15. Matthews GVT (1961) "Nonsense" orientation in mallard Anas Platyrhynchos and its relation to experiments on bird navigation. Ibis 103a: 211-230.

16. Schmidt-Koenig K (1964) Uber die orientierung der vogel; Experimente und probleme. Naturwissenschaften 51: 423-431.

17. Bruderer $B$ (1975) Variation in direction and spread of directions of bird migration in northern Switzerland. Der Ornithologische Beobachter 72: 169-179.

18. Edward B (1981) Circular Statistics in Biology. Academic Press, London.

19. Fisher NI (1993) Statistical analysis of circular data. Cambridge University press, Melbourne.

20. Umbach D, Jammalamadaka SR (2009) Building asymmetry into circular distributions. Stat Probab Lett 79: 659-663. 